\title{
Cryoballoon or Radiofrequency Ablation for Atrial Fibrillation
}

TO THE EDITOR: The ambitious multicenter, randomized, controlled trial by Kuck et al. (June 9 issue) ${ }^{1}$ showed that cryoballoon ablation was noninferior to radiofrequency ablation in paroxysmal atrial fibrillation. Beyond this central point, the trial raised two interesting issues. First, not only was the mean total procedure time shorter with cryoballoon ablation than with radiofrequency ablation (124 minutes vs. 141 minutes), but the standard deviation was much narrower (39 minutes vs. 55 minutes), findings that indicate that cryoballoon ablation is a more predictable procedure than radiofrequency ablation. ${ }^{2}$

Second, patients treated with catheters using contact-force sensing had a rate of the primary efficacy end point (including recurrence of atrial fibrillation) that was approximately 10 percentage points higher than patients treated with radiofrequency catheters not using contact-force sensing, a finding that contrasts with recent data. ${ }^{3}$ However, the lack of surround-flow irrigation technology with contact-force sensing (vs. the presence of surround flow in standard radiofrequency ablation) could have played a role. ${ }^{4,5}$

It would be of interest to know the consistency of outcomes among centers according to caseload in the two treatment groups. In spite of possible confounders, especially sample power, this could provide us with insights into two questions: Is cryoballoon ablation truly noninferior to radiofrequency ablation even in the most experienced centers? And did the use of surround flow compensate for the lack of contactforce sensing?

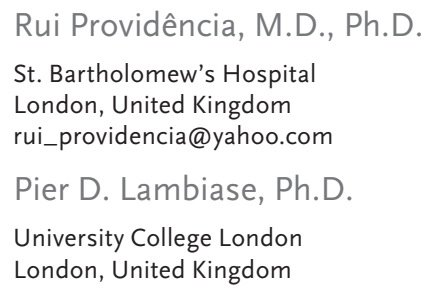

No potential conflict of interest relevant to this letter was reported.

1. Kuck K-H, Brugada J, Fürnkranz A, et al. Cryoballoon or radiofrequency ablation for paroxysmal atrial fibrillation. $\mathrm{N}$ Engl $\mathrm{J}$ Med 2016;374:2235-45.

2. Providencia R, Defaye P, Lambiase PD, et al. Results from a multicentre comparison of cryoballoon vs. radiofrequency ablation for paroxysmal atrial fibrillation: is cryoablation more reproducible? Europace 2016 June 5 (Epub ahead of print).

3. Marijon E, Fazaa S, Narayanan K, et al. Real-time contact force sensing for pulmonary vein isolation in the setting of paroxysmal atrial fibrillation: procedural and 1-year results. J Cardiovasc Electrophysiol 2014;25:130-7.

4. Bertaglia E, Fassini G, Anselmino M, et al. Comparison of ThermoCool Surround Flow catheter versus ThermoCool catheter in achieving persistent electrical isolation of pulmonary veins: a pilot study. J Cardiovasc Electrophysiol 2013;24:269-73.

5. Sciarra L, Golia P, Natalizia A, et al. Which is the best catheter to perform atrial fibrillation ablation? A comparison between standard ThermoCool, SmartTouch, and Surround Flow catheters. J Interv Card Electrophysiol 2014;39:193-200.

DOI: 10.1056/NEJMc1609160

TO THE EDITOR: Kuck et al. provide a comprehensive report on adverse events after either radiofrequency ablation or cryoballoon ablation (Table 3 in their article). However, we are concerned that they have left out an underappreciated and potentially devastating procedure-related complication - namely, gastroparesis. We recently described a patient who had severe gastroparesis, evidenced by a gastric-emptying study showing $86 \%$ of retained gastric contents 4 hours after ingestion, immediately after a cryoablation procedure for atrial fibrillation. ${ }^{1}$

Gastroparesis after ablation procedures for atrial fibrillation may be related to injury to the periesophageal vagus nerve; the vagus nerve innervates the stomach and pylorus and runs in close proximity to the inferior pulmonary veins and left atrium. ${ }^{2}$ A case series of 104 patients who underwent either cryoballoon ablation or radiofrequency ablation estimates the prevalence of postprocedural gastroparesis to be $10 \%$ and $2 \%$, respectively. ${ }^{2}$ We are curious whether enrolled patients had gastroparesis after ablation and whether the authors can comment on this procedural complication in the context of their recent trial.

Jonathan S. Zipursky, M.D.
University of Toronto
Toronto, ON, Canada
jonathan.zipursky@mail.utoronto.ca
Steven Shadowitz, M.D.C.M.
Sunnybrook Health Sciences Centre
Toronto, ON, Canada
No potential conflict of interest relevant to this letter was re-
ported.
1. Lee DS, Lee SJ. Severe gastroparesis following radiofrequency
catheter ablation for atrial fibrillation: suggestion for diagnosis,


treatment, and device for gastroparesis after RFCA. Case Rep Gastrointest Med 2014;2014:923637.

2. Aksu T, Golcuk S, Guler TE, Yalin K, Erden I. Gastroparesis as a complication of atrial fibrillation ablation. Am J Cardiol 2015; 116:92-7.

DOI: 10.1056/NEJMc1609160

TO THE EDITOR: The FIRE AND ICE trial concluded that cryoballoon ablation was not inferior to radiofrequency ablation of pulmonary veins for atrial fibrillation. However, no anatomical variations in pulmonary veins were considered in that trial. We wish to highlight that the anatomical features of pulmonary veins may influence the efficacy of pulmonary-vein isolation and should be taken into account when selecting the ablation method. ${ }^{1}$ Only 47 to $81 \%$ of hearts have four standard pulmonary veins. ${ }^{2,3}$ An additional, separate ostium of the middle right pulmonary vein is the most common variation and is present in approximately $20 \%$ of hearts. Because this vein also has the myocardial sleeve, clinicians should be aware of this anomaly. ${ }^{3}$ Moreover, the mean $( \pm S D$ ) diameter of this vein $(8.2 \pm 4.1 \mathrm{~mm})^{3}$ is typically too small for a cryoballoon, so with this method the middle right pulmonary vein may easily be omitted. The radiofrequency catheter ablation system may be a better option in atypical pulmonary-vein patterns; the middle right pulmonary vein ostium may even be unintentionally isolated when performing a pulmonary-vein ablation on the right side. The individual pulmonary-vein pattern should always be imaged before ablation to select the appropriate technique and achieve maximum efficacy. ${ }^{4}$

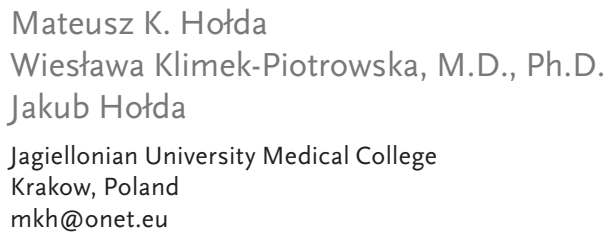

No potential conflict of interest relevant to this letter was reported.

1. Güler E, Güler GB, Demir GG, et al. Effect of pulmonary vein anatomy and pulmonary vein diameters on outcome of cryoballoon catheter ablation for atrial fibrillation. Pacing Clin Electrophysiol 2015;38:989-96.

2. McLellan AJ, Ling LH, Ruggiero D, et al. Pulmonary vein isolation: the impact of pulmonary venous anatomy on longterm outcome of catheter ablation for paroxysmal atrial fibrillation. Heart Rhythm 2014;11:549-56.

3. Klimek-Piotrowska W, Hołda MK, Piątek K, Koziej M, Hołda J. Normal distal pulmonary vein anatomy. PeerJ 2016;4:e1579.

4. Porres DV, Morenza OP, Pallisa E, Roque A, Andreu J, Mar- tínez M. Learning from the pulmonary veins. Radiographics 2013;33:999-1022.

DOI: $10.1056 /$ NEJMc1609160

THE AUTHORS REPLY: In response to Providência et al.: the FIRE AND ICE trial had requirements to ensure that centers were experienced in both cryoballoon ablation and radiofrequency current ablation. Specifically, each center had to provide at least one investigator who was proficient in both techniques ( $\geq 50$ cases with each technology), and before the use of advanced-generation catheters, each center had to complete at least 10 cases. A test of center effect on the primary efficacy analysis revealed no significant differences $(P=0.83){ }^{1}$ With respect to the radiofrequency catheters used in the trial, there were 46 surround-flow catheters and 93 contact-force-sensing catheters. There were no contact-force-sensing surroundflow radiofrequency catheters, because enrollment was completed in January 2015, and Conformité Européenne (CE)-mark approval was received in May 2014, which did not allow centers time to finish the minimum number of required cases. Importantly, this trial was not powered to investigate further differences between generations of catheters.

In response to Zipursky and Shadowitz: the trial physicians reported no instance of gastroparesis, but there were postprocedural adverse events that had symptoms that are shared with gastroparesis (abdominal pain, diabetic gastroparesis, epigastric discomfort, gastritis, impaired gastric emptying, nausea, and vomiting) (Table S4 in the Supplementary Appendix of the article, available at NEJM.org). For the incidence of these combined events, no significant difference between groups was present (cryoballoon ablation, $3.2 \%$ [12 of 374 patients]; radiofrequency ablation, $2.1 \%$ [8 of 376 patients]; $\mathrm{P}=0.38$ ). However, the study by Aksu et al. showed that gastroparesis had resolved in all patients who received cryoballoon ablation, whereas one patient who received radiofrequency ablation had persistent gastroparesis at the 6-month follow-up visit, findings that suggest that the energy source may influence the severity of collateral tissue damage. ${ }^{2}$

In response to Hołda et al.: in the FIRE AND ICE trial, all anatomical pulmonary-vein variations (present at enrollment) were allowed. In fact, the only exclusion criteria regarding pulmonaryvein anatomical features was that patients were 
excluded for right-sided pulmonary-vein diameters greater than $26 \mathrm{~mm}$. In the trial, $98.9 \%$ of all pulmonary veins were isolated at the index procedure in the cryoballoon group, as compared with $97.9 \%$ in the radiofrequency group. In the cryoballoon group, $100 \%$ of the left common pulmonary veins (28 of 28 ) and $92 \%$ of the right middle pulmonary veins (12 of 13) were isolated at the index procedure. In the radiofrequency group, $77 \%$ of the left common pulmonary veins (30 of 39) and $48 \%$ of the right middle pulmonary veins (11 of 23) were isolated at the index ablation.

Currently, prescreening of pulmonary-vein anatomical features is not required. More important, the cryoballoon group has shown significantly fewer reinterventions and rehospitalizations than the radiofrequency group, ${ }^{3}$ and these patient-relevant disease-burden characteristics should be considered when making the decision about what type of catheter ablation should be performed.
Karl-Heinz Kuck, M.D.

Asklepios Klinik St. Georg

Hamburg, Germany

k.kuck@asklepios.com

Josep Brugada, M.D.

Hospital Clínic de Barcelona

Barcelona, Spain

Jean-Paul Albenque, M.D.

Clinique Pasteur

Toulouse, France

Since publication of their article, the authors report no further potential conflict of interest.

1. Kuck KH, FIRE AND ICE Investigators. Cryoballoon or radiofrequency ablation for paroxysmal atrial fibrillation: the FIRE AND ICE trial. Presented at the American College of Cardiology Annual Scientific Session, Chicago, April 4, 2016.

2. Aksu T, Golcuk S, Guler TE, Yalin K, Erden I. Gastroparesis as a complication of atrial fibrillation ablation. Am J Cardiol 2015;116:92-7.

3. Kuck KH, Fürnkranz A, Chun KR, et al. Cryoballoon or radiofrequency ablation for symptomatic paroxysmal atrial fibrillation: reintervention, rehospitalization, and quality-of-life outcomes in the FIRE AND ICE trial. Eur Heart J 2016 July 5 (Epub ahead of print).

DOI: 10.1056/NEJMc1609160

\section{Evidence for Transmission of Zika Virus by Platelet Transfusion}

TO THE EDITOR: Zika virus (ZIKV) is a mosquitoborne virus that has important secondary means of transmission that include perinatal and sexual modes. ${ }^{1-3}$ The potential for transmission in transfused donated blood components has been a concern owing to the detection of ZIKV viremia in healthy blood donors. ${ }^{4}$

This report from Brazil describes two cases of likely ZIKV transmission by blood transfusion from one presymptomatic infected person who donated platelets by apheresis on January 16, 2016. The two leukodepleted platelet units were irradiated with 25 Gy delivered by an IBL-437C gamma irradiator (Cis Bio International) and were transfused in different patients on January 19 (day 0). On January 21, the donor called the blood bank to report a cutaneous rash, retroorbital pain, and pain in both knees that had begun on January 18. An investigation was initiated under the hospital's clinical protocol for transfusion-associated adverse events, with the donor and both patients providing written informed consent.

Two samples that were obtained from the donor before and after donation were negative for chikungunya virus (CHIKV) and dengue virus (DENV) on reverse-transcriptase-polymerasechain-reaction (RT-PCR) assay, but the index plasma and urine samples 14 days later were positive for ZIKV (Table 1). (Details of the methods that were used and results are provided in the Supplementary Appendix, available with the full text of this letter at NEJM.org.) Serologic analysis by means of point-of-care testing, inhouse indirect immunofluorescence assay (IFA), and plaque-reduction neutralization testing (PRNT) confirmed the presence of acute ZIKV infection in the donor.

The first recipient (Patient 1) was a 54-yearold woman with the primary myelofibrosis syndrome. The second recipient (Patient 2) was a 14-year-old girl with acute myeloid leukemia who had undergone haploidentical bone marrow transplantation on January 6, after which she had been receiving continuous immunosuppressive therapy. Routine pretransfusion samples obtained from the two patients were negative on PCR assay for CHIKV, DENV, and ZIKV, but samples collected 6 days after platelet transfusion in Patient 1 and 23 to 51 days after platelet transfu- 